\title{
Shear instabilities in the dust layer of the solar nebula I. The linear analysis of a non-gravitating one-fluid model without the Coriolis and the solar tidal forces
}

\author{
Minoru Sekiya ${ }^{1}$ and Naoki Ishitsu ${ }^{2}$ \\ ${ }^{1}$ Department of Earth and Planetary Sciences, Faculty of Sciences, Kyushu University, 6-10-1 Hakozaki, Fukuoka 812-8581, Japan \\ ${ }^{2}$ Department of Earth and Planetary Sciences, Graduate School of Sciences, Kyushu University, 6-10-1 Hakozaki, Fukuoka 812-8581, Japan
}

(Received December 3, 1999; Revised May 25, 2000; Accepted June 13, 2000)

\begin{abstract}
As dust aggregates settled toward the midplane of the solar nebula, a thin dust layer was formed. The rotational velocity was a function of the distance from the midplane in this layer, and the shear induced turbulence might occur, which prevented the dust aggregates from settling further toward the midplane. Thus, it was difficult for the dust density on the midplane to exceed the critical density of the gravitational stability. In this paper, the linear analysis of the shear instability is made under the following assumptions: The self-gravity, the solar tidal force (thus the Keplerian shear), and the Coriolis force are neglected; the unperturbed state has a constant Richardson's number in the dust layer; further we restrict ourselves to the case where dust aggregates are small enough, and a mixture of dust and gas is treated as one fluid. Numerical results show that the growth rate of the most unstable mode is much less than the Keplerian angular frequency, as long as the Richardson number is larger than 0.1 .
\end{abstract}

\section{Introduction}

Dust grains which had been homogeneously distributed initially in the solar nebula stuck together and settled toward the midplane and a thin dust layer was formed as long as the nebula was laminar (Safronov, 1969; Weidenschilling, 1980; Nakagawa et al., 1981 and 1986). If the midplane dust density exceeded the critical density, planetesimals were formed due to gravitational instabilities (Safronov, 1969; Goldreich and Ward, 1973; Coradini et al., 1981; Sekiya, 1983). However, recent investigations raised suspicion on this scenario (Weidenschilling, 1980 and 1984; Cuzzi et al., 1993; Weidenschilling and Cuzzi, 1993; Champney et al., 1995; Sekiya, 1998). The rotational velocity of a nebular fluid element was a bit smaller than the circular Keplerian velocity due to the radial gas pressure gradient in the solar nebula. As dust aggregates settled toward the midplane, the rotational velocity increased around the midplane because of the relative ineffectiveness of the radial pressure gradient compared to the centrifugal force and the solar gravity, since the latter two forces are proportional to the mass. The rotational velocity was then a function of the distance from the midplane. Thus the shear induced turbulence might occur, and the turbulence might prevent the dust aggregates from settling further and the dust density could not exceed the critical value of the gravitational stability. If this was the case, planetesimals could not be formed by gravitational instability; they should be formed by mutual sticking of dust aggregates due to non-gravitational forces, e.g. the van der Waals force. It is important to elucidate the physics of the shear instabilities in the dust layer, in order to know the real

Copy right (C) The Society of Geomagnetism and Earth, Planetary and Space Sciences (SGEPSS); The Seismological Society of Japan; The Volcanological Society of Japan; The Geodetic Society of Japan; The Japanese Society for Planetary Sciences. formation story of planetesimals.

We plan to work on the stability problems of the dust layer in series of papers. This paper is the first one which makes the linear analysis of the shear instability in the dust layer with the simplest assumptions: (1) The self-gravity is neglected. (2) A mixture of gas and dust is treated as one fluid, which is a good approximation in the case where dust aggregate sizes are small ( $\lesssim 1 \mathrm{~cm})$. (3) The solar tidal force, which is the sum of the radial component of solar gravity and the centrifugal force, is neglected; thus the radial shear $\partial v / \partial r$ is not incorporated in the unperturbed state, and only $z$-component of the solar gravity is taken into account, where $v$ is the rotational velocity of a fluid which is a mixture of gas and dust, $r$ is the distance from the rotation axis, and $z$ is a coordinate perpendicular to the midplane of the solar nebula ( $z=0$ on the midplane). (4) The effects of the Coriolis force are neglected. (5) The effects of the radial density and pressure gradients of the unperturbed state are only incorporated in the unperturbed rotational velocity distribution $v_{0}(z)$. (6) Local Cartesian coordinates $(x, y, z)$ are used and we neglect the curvature of a circle with constant values of $r$ and $z$. (7) The unperturbed state has a constant Richardson's number [see Eq. (13)] in the dust layer. In subsequent papers, these assumptions would be modified.

\section{Basic Equations}

If dust aggregates are small enough to couple firmly to gas and a mixture of dust and gas is treated as one fluid, the stationary rotation velocity in the dust layer is given by (see e.g. Sekiya, 1998)

$$
v=\left[1-\left(\rho_{g} / \rho\right) \eta(r)\right] v_{K}(r),
$$

where $\rho_{g}$ is the gas density and $\rho$ is the total (dust plus gas) density, which is equal to $\rho_{g}+\rho_{d}$ ( $\rho_{d}$ being the dust density), 


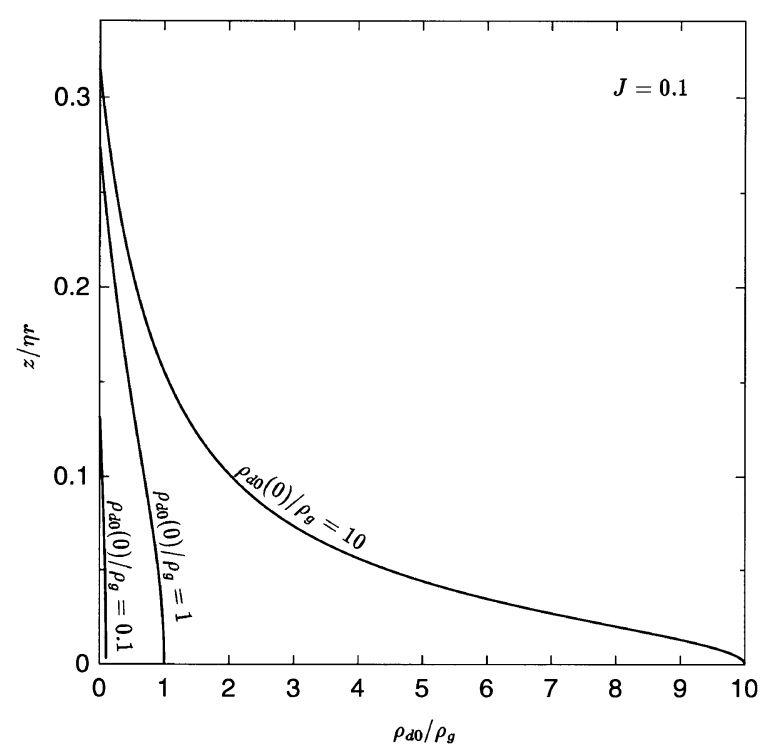

Fig. 1. The distributions of the unperturbed dust density $\rho_{d 0}$ given by Eqs. (14) and (4), in the cases where $\rho_{d 0}(0) / \rho_{g}=0.1,1$ and 10 with $J=0.1$.

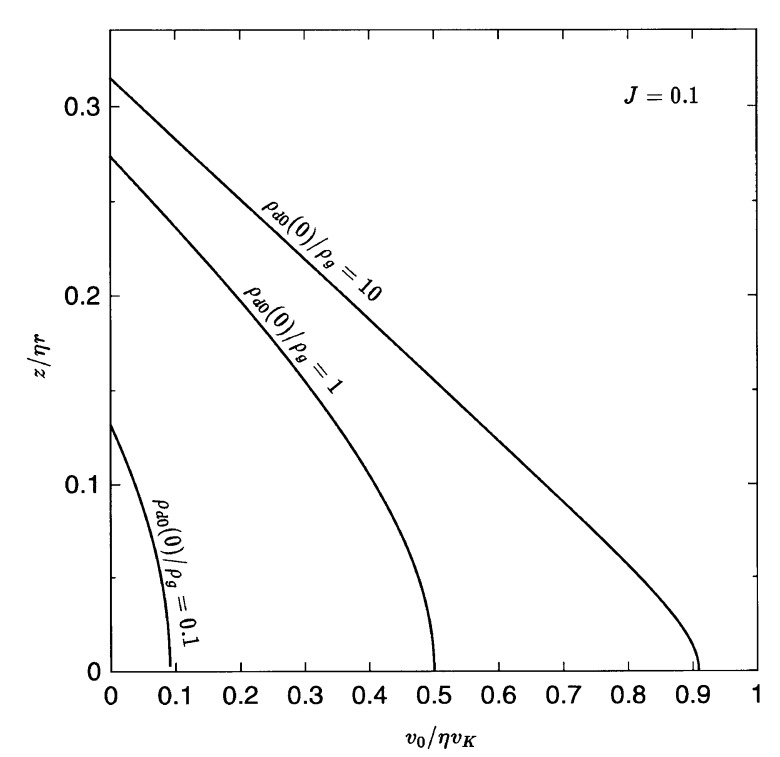

Fig. 2. The distributions of the unperturbed azimuthal velocity $v_{0}$, given by Eq. (3), in the cases where $\rho_{d 0}(0) / \rho_{g}=0.1,1$ and 10 with $J=0.1$.

$v_{K}(r)$ is the circular Keplerian velocity with radius $r$ from the sun, and

$$
\eta(r)=-(\partial P / \partial \ln r) /\left\{2\left[v_{K}(r)\right]^{2} \rho_{g}\right\},
$$

where $P$ is the gas pressure. Eqs. (1) and (2) show that a shear flow $\partial v / \partial z$ arises due to gradient of dust to gas ratio $\partial\left(\rho / \rho_{g}\right) / \partial z=\left[\partial\left(\rho_{d} / \rho_{g}\right) / \partial z\right]$, which, in turn, is caused by settling of dust aggregates toward the midplane.

In the following, we consider a local region in the dust layer around $r=r_{0}$ and use the Cartesian coordinate system $(x, y, z)$, whose origin is on the midplane at $r=r_{0}$, and which rotates around the sun with the velocity $v=(1-\eta) v_{K}$, i.e. the velocity of gas dominant region (where $\rho=\rho_{g}$ ) at $r=r_{0}$, and $x$ and $y$ stand for the radial and the azimuthal di-

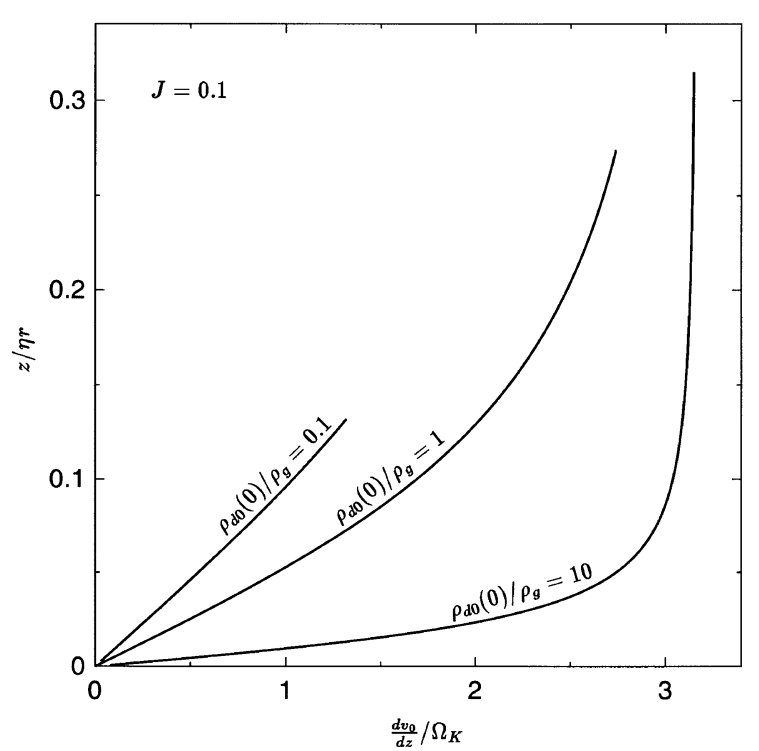

Fig. 3. The distributions of vertical gradient of the unperturbed azimuthal velocity $d v_{0} / d z$ given by Eq. (5) in the cases where $\rho_{d 0}(0) / \rho_{g}=0.1,1$ and 10 with $J=0.1$.

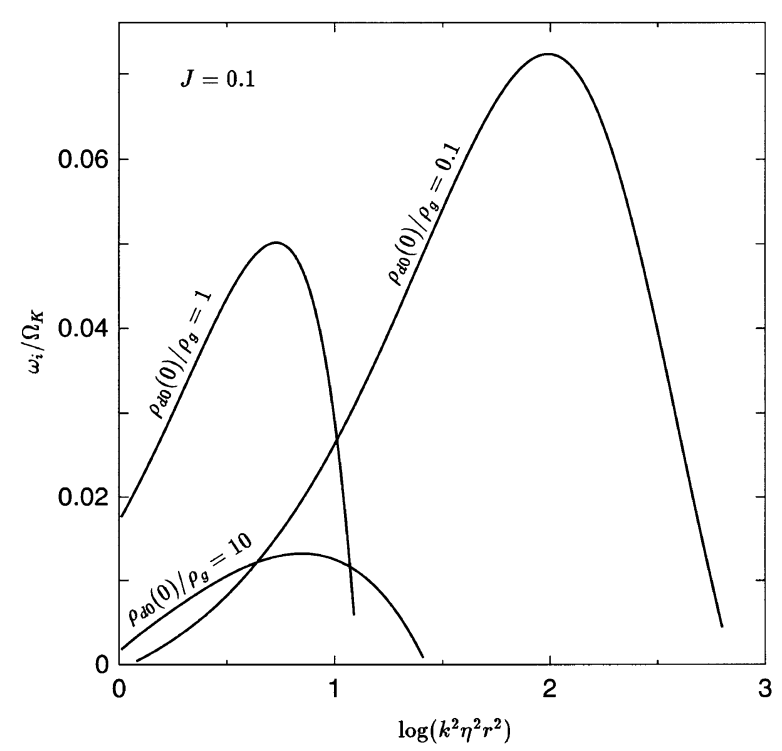

Fig. 4. The growth rates of the instability $\omega_{i}$ as functions of the azimuthal wave number $k$ in the cases where $\rho_{d 0}(0) / \rho_{g}=0.1,1$ and 10 with $J=0.1$.

rections, respectively [hereafter we write $r, \eta$ and $v_{K}$ instead of $r_{0}, \eta\left(r_{0}\right)$ and $v_{K}\left(r_{0}\right)$, respectively, for simplicity]. For the first step to solve this problem, we simplify the problem assuming that the Coriolis force and the solar tidal force are neglected, and that the unperturbed fluid is non-rotating with respect to $z$-axis and has a shear motion only depending on $z$-direction

$$
v_{0}=\left\{1-\left[\rho_{g} / \rho_{0}(z)\right]\right\} \eta v_{K},
$$

where $\rho_{0}(z)$ is the unperturbed total (gas plus dust) density:

$$
\rho_{0}(z)=\rho_{g}+\rho_{d 0}(z)
$$

where $\rho_{d 0}$ is the unperturbed dust density. Note that Eq. (3) differs from Eq. (1), since we use here a frame of reference 


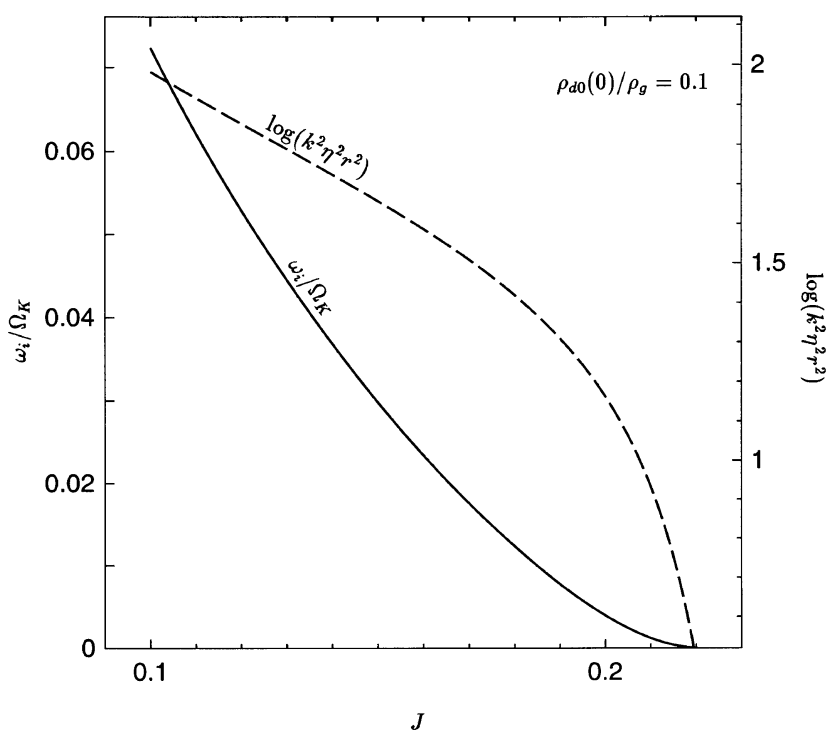

Fig. 5. The growth rate $\omega_{i}$ of the mode with the most unstable wave number as a function of the Richardson number $J$ in the case where $\rho_{d 0}(0) / \rho_{g}=0.1$. The most unstable wave number squared is shown by the dashed curve.

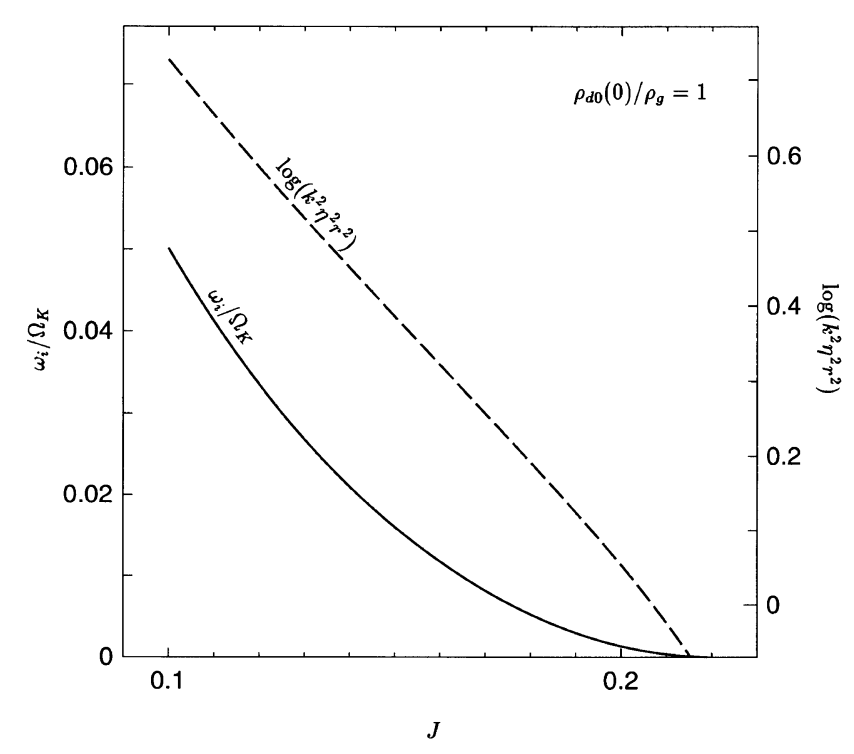

Fig. 6. Same as Fig. 5 except that $\rho_{d 0}(0) / \rho_{g}=1$.

moving with $v=(1-\eta) v_{K}$. We assume that the gas density $\rho_{g}$ is constant, which is a good approximation for a thin dust layer (Sekiya, 1983). Then the shear rate is given by

$$
d v_{0} / d z=\rho_{g}\left[\rho_{0}(z)\right]^{-2}\left(d \rho_{0} / d z\right) \eta v_{K}
$$

A mixture of gas and dust behaves as one incompressible fluid in the region around the midplane, as long as dust aggregates are small enough (Sekiya, 1998). Thus the equations of continuity and incompressibility are written

$$
\begin{aligned}
& \frac{\partial u}{\partial x}+\frac{\partial v}{\partial y}+\frac{\partial w}{\partial z}=0 \\
& \frac{\partial \rho}{\partial t}+u \frac{\partial \rho}{\partial x}+v \frac{\partial \rho}{\partial y}+w \frac{\partial \rho}{\partial z}=0 .
\end{aligned}
$$

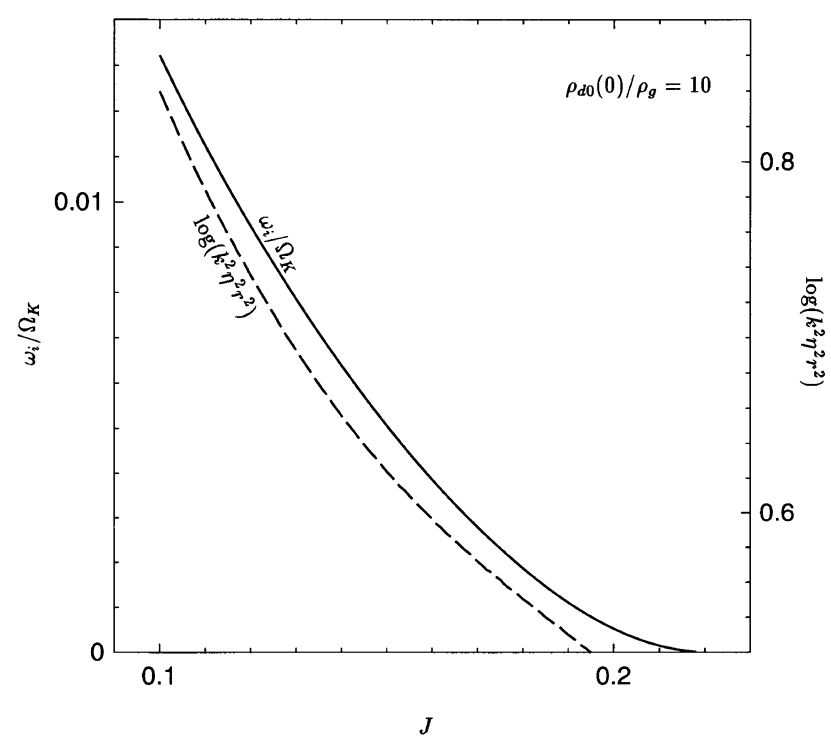

Fig. 7. Same as Fig. 5 except that $\rho_{d 0}(0) / \rho_{g}=10$.

We only take $z$-component of the solar gravity $-\Omega_{K}^{2} z$ into account, where $\Omega_{K}$ is the circular Keplerian angular velocity at $r_{0}$. The Euler equations are written

$$
\begin{aligned}
& \frac{\partial u}{\partial t}+u \frac{\partial u}{\partial x}+v \frac{\partial u}{\partial y}+w \frac{\partial u}{\partial z}=-\frac{1}{\rho} \frac{\partial P}{\partial x} \\
& \frac{\partial v}{\partial t}+u \frac{\partial v}{\partial x}+v \frac{\partial v}{\partial y}+w \frac{\partial v}{\partial z}=-\frac{1}{\rho} \frac{\partial P}{\partial y} \\
& \frac{\partial w}{\partial t}+u \frac{\partial w}{\partial x}+v \frac{\partial w}{\partial y}+w \frac{\partial w}{\partial z}=-\frac{1}{\rho} \frac{\partial P}{\partial z}-\Omega_{K}^{2} z
\end{aligned}
$$

where $(u, v, w)$ are the $(x, y, z)$ components of the velocity, respectively. Note that the solar tidal force is neglected in Eq. (8), and the Coriolis force is neglected in Eqs. (8) and (9) in this paper.

The unperturbed velocity is given by Eq. (3) and

$$
u_{0}=w_{0}=0 \text {. }
$$

The unperturbed pressure $P_{0}$ is given by

$$
\frac{1}{\rho_{0}} \frac{d P_{0}}{d z}=-\Omega_{K}^{2} z .
$$

Note that $P_{0}$ is assumed to be independent of $x$ in the simplified model of this paper, although the shear motion $d v_{0} / d z$ arises due to the radial pressure gradient through $\eta$ given by Eq. (2) in the original problem. We solve in this paper a problem in non-rotating system with shear $d v_{0} / d z$ given by Eq. (5) and only $z$-component of the solar gravity.

The stability of a shearing parallel flow is characterized by the Richardson number (Chandrasekhar, 1961; Howard, 1961):

$$
\begin{aligned}
J & =-\left[\partial \rho_{0}(z) / \partial z\right] \rho_{0}(z)^{-1} \Omega_{K}^{2} z\left[\partial v_{0}(z) / \partial z\right]^{-2} \\
& =-z\left[\rho_{0}(z)\right]^{3}\left[d \rho_{0} / d z\right]^{-1}\left(\rho_{g} \eta r\right)^{-2}
\end{aligned}
$$

where Eq. (5) is used. A flow is stable as long as $J \geq 1 / 4$ (Chandrasekhar, 1961; Howard, 1961). 


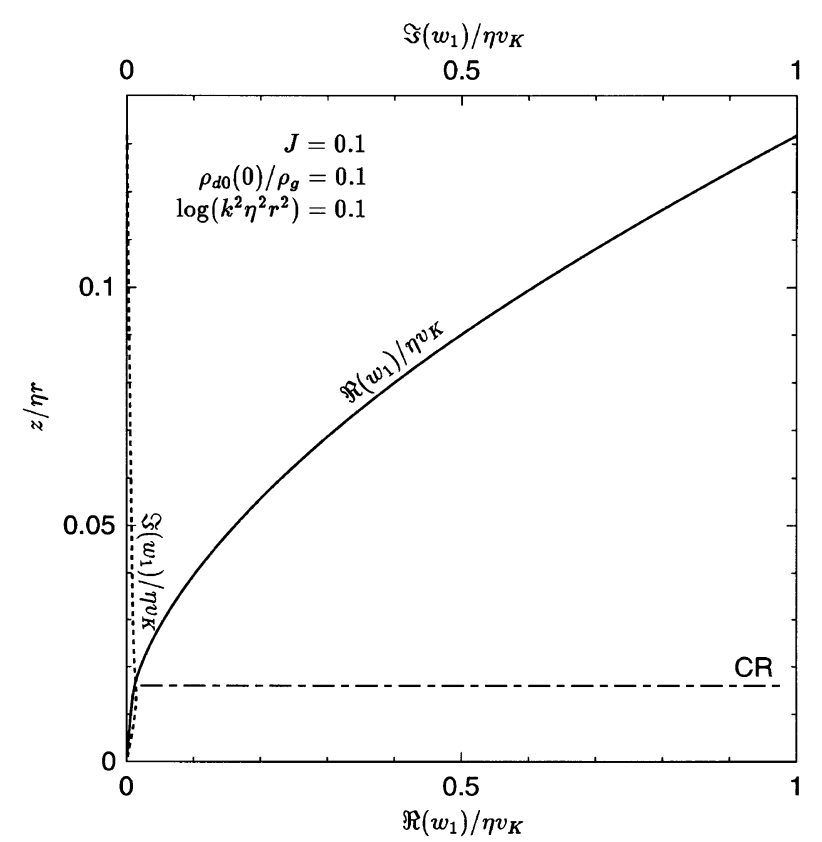

Fig. 8. The real and imaginary parts of the eigenfunction $\Re\left(w_{1}\right)$ and $\Im\left(w_{1}\right)$, respectively, of Eq. (22) in the case where $\log \left(k^{2} \eta^{2} r^{2}\right)=0.1$, with $J=0.1$ and $\rho_{d 0}(0) / \rho_{g}=0.1$. The co-rotation point is shown by a dash-dotted line with characters "CR".

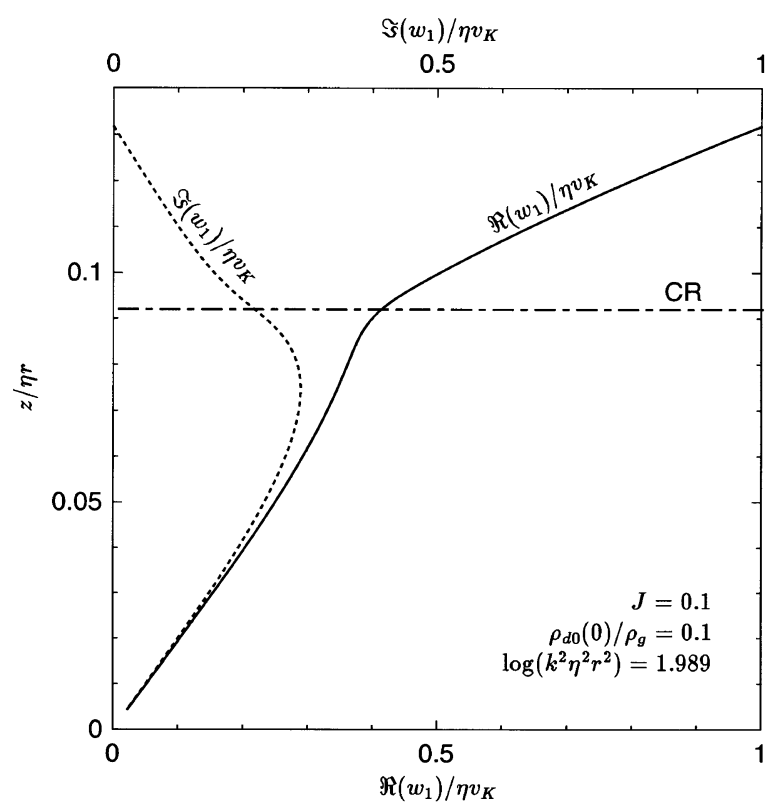

Fig. 9. Same as Fig. 8 except that $\log \left(k^{2} \eta^{2} r^{2}\right)=1.989$.

A detailed distribution of the Richardson number in the nebula depends on various processes: dust sticking, fractal structure of dust aggregates, turbulence in the solar nebula, etc. Time dependent calculations should be made in future to know the evolution of the nebula, after basic processes, e.g. shear induced turbulence, dust sticking etc., would be elucidated. However, we here assume that an unperturbed state has a constant Richardson's number in the dust layer for simplicity, and parameterize the dust settling by decrease of the Richardson number. If the self-gravity of the dust

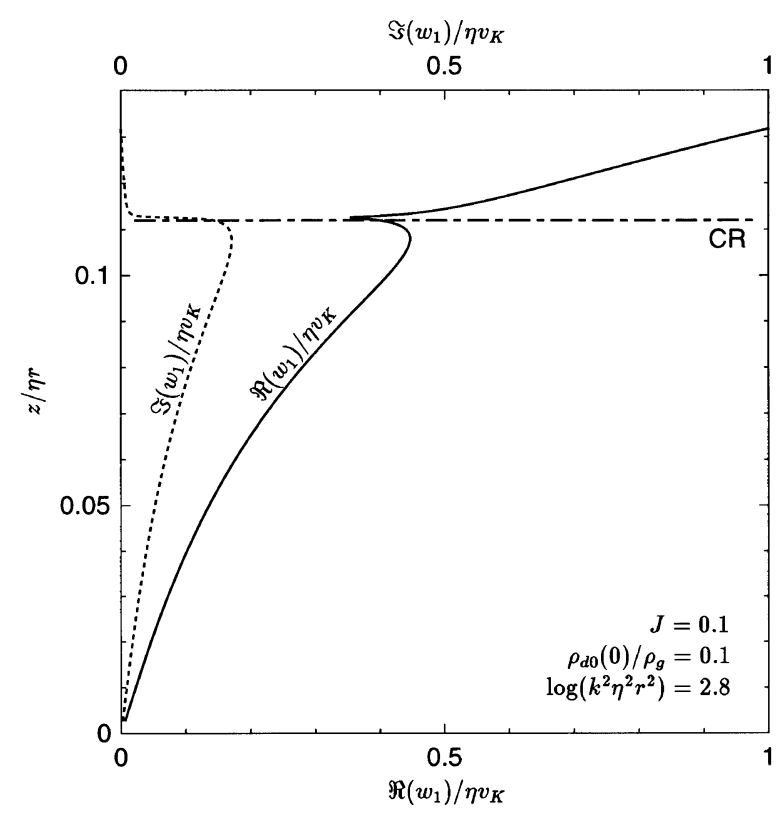

Fig. 10. Same as Fig. 8 except that $\log \left(k^{2} \eta^{2} r^{2}\right)=2.8$.

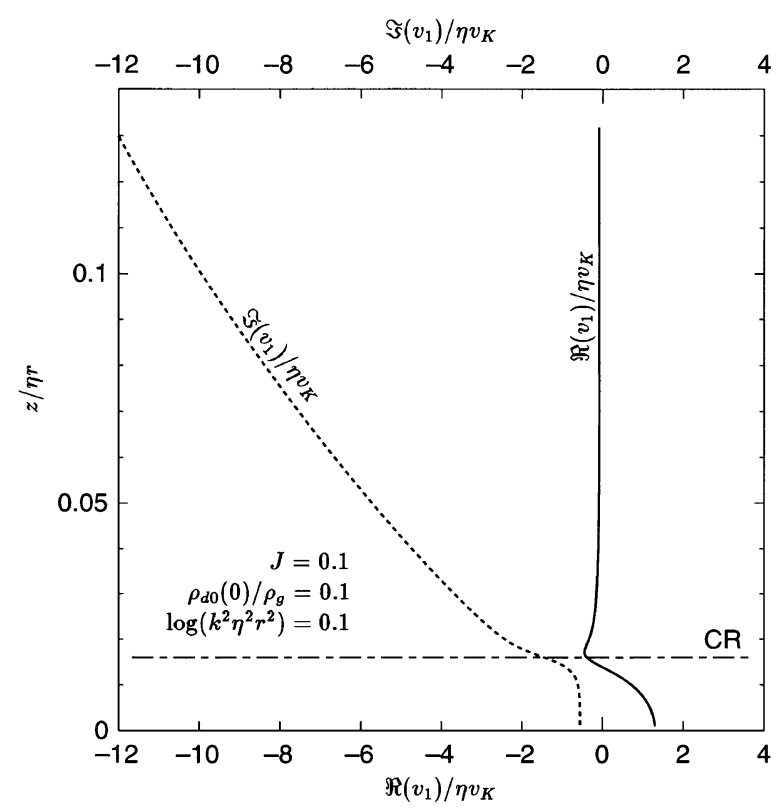

Fig. 11. The real and imaginary parts of $v_{1}$, which are calculated from Eq. (16) with $k_{x}=0$, in the case where $\log \left(k^{2} \eta^{2} r^{2}\right)=0.1$, with $J=0.1$ and $\rho_{d 0}(0) / \rho_{g}=0.1$. The co-rotation point is shown by a dash-dotted line with characters "CR".

layer is sufficiently small, the total density distribution with a constant Richardson's number $J$ is given by Sekiya (1998):

$$
\rho_{0}= \begin{cases}\rho_{g} / \sqrt{\left[z^{2} /\left(J \eta^{2} r^{2}\right)\right]+\left[\rho_{g} / \rho_{0}(0)\right]^{2}} & \text { for }|z|<z_{d}, \\ \rho_{g} & \text { for }|z| \geq z_{d} .\end{cases}
$$

Here $z_{d}=\sqrt{J} \eta r \sqrt{1-\left[\rho_{g} / \rho_{0}(0)\right]^{2}}$ is the half thickness of the dust layer, and $\rho_{0}(0)$ is the unperturbed total density on the midplane, which is determined from

$$
\Sigma_{d}=2 \int_{0}^{z_{d}} \rho_{d 0} d z
$$




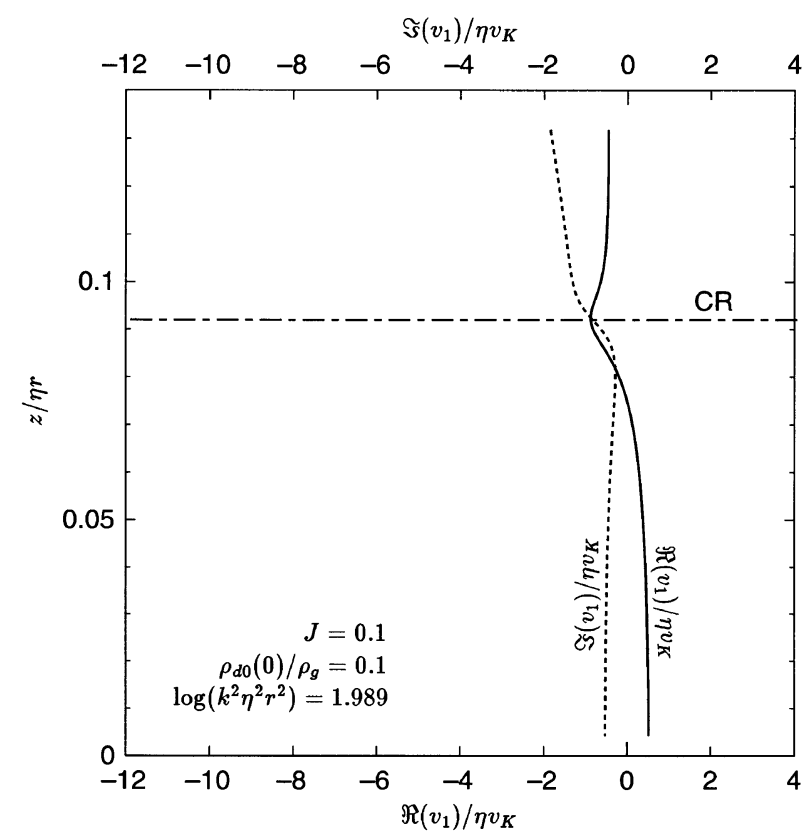

Fig. 12. Same as Fig. 11 except that $\log \left(k^{2} \eta^{2} r^{2}\right)=1.989$.

where $\Sigma_{d}$ is the dust column density, and $\rho_{d 0}$ is calculated from Eq. (4).

Perturbation equations are written assuming perturbed quantities are proportional to $\exp \left(i k_{x} x+i k_{y} y-i \omega t\right)$ :

$$
\begin{aligned}
& i k_{x} u_{1}+i k_{y} v_{1}+\frac{d w_{1}}{d z}=0, \\
& -i \bar{\omega} \rho_{1}+\frac{d \rho_{0}}{d z} w_{1}=0, \\
& -i \bar{\omega} u_{1}=-\frac{i k_{x} P_{1}}{\rho_{0}}, \\
& -i \bar{\omega} v_{1}+\frac{d v_{0}}{d z} w_{1}=-\frac{i k_{y} P_{1}}{\rho_{0}}, \\
& -i \bar{\omega} w_{1}=-\frac{1}{\rho_{0}} \frac{d P_{1}}{d z}-\frac{\Omega_{K}^{2} z}{\rho_{0}} \rho_{1},
\end{aligned}
$$

where characters with subscript 1 designate perturbed quantities, and $\bar{\omega} \equiv \omega-k_{y} v_{0}=-k_{y} \bar{v}, \bar{v} \equiv v_{0}-c$, and $c \equiv \omega / k_{y}$. From Eqs. (16), (18) and (19), we have

$$
P_{1}=\frac{i \rho_{0}}{k^{2}}\left(\bar{\omega} \frac{d w_{1}}{d z}+k_{y} \frac{d v_{0}}{d z} w_{1}\right),
$$

where $k=\left(k_{x}^{2}+k_{y}^{2}\right)^{1 / 2}$. Substituting Eqs. (17) and (21) into Eq. (20), we have

$$
\begin{aligned}
\frac{d^{2} w_{1}}{d z^{2}} & +\frac{1}{\rho_{0}} \frac{d \rho_{0}}{d z} \frac{d w_{1}}{d z}-\left(k^{2}+\frac{1}{\bar{v}} \frac{d^{2} v_{0}}{d z^{2}}\right. \\
& \left.+\frac{1}{\rho_{0}} \frac{d \rho_{0}}{d z} \frac{1}{\bar{v}} \frac{d v_{0}}{d z}+\frac{k^{2}}{k_{y}^{2}} \frac{\Omega_{K}^{2} z}{\bar{v}^{2}} \frac{1}{\rho_{0}} \frac{d \rho_{0}}{d z}\right) w_{1}=0
\end{aligned}
$$

In regions outside the dust layer $|z| \geq z_{d}$, Eqs. (21) and (22) are simplified as

$$
P_{1}=\frac{i \rho_{g} \bar{\omega}}{k^{2}} \frac{d w_{1}}{d z}
$$

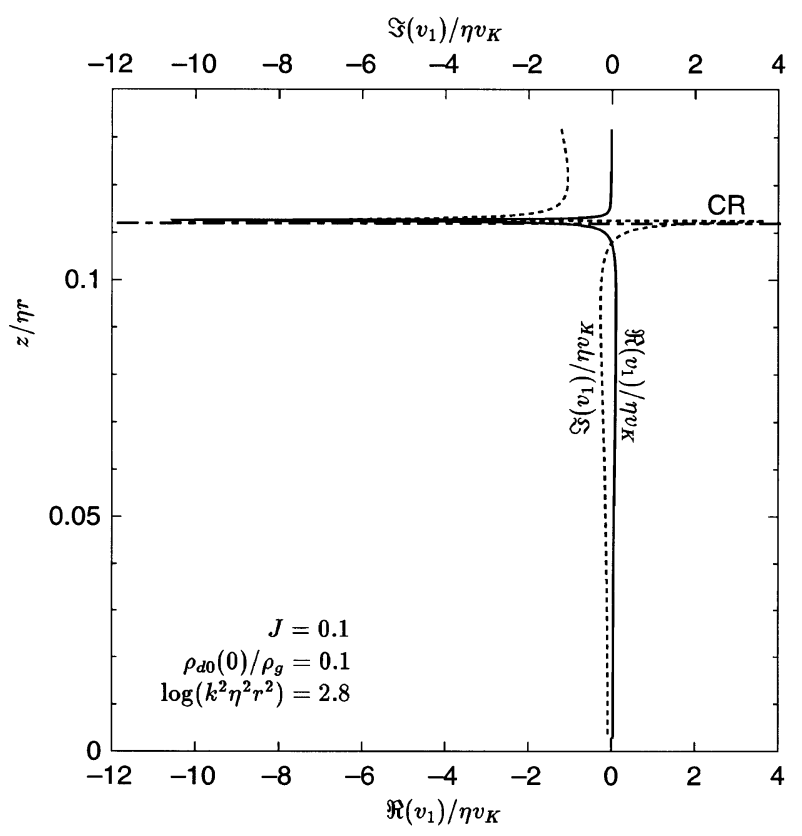

Fig. 13. Same as Fig. 11 except that $\log \left(k^{2} \eta^{2} r^{2}\right)=2.8$.

and

$$
\frac{d^{2} w_{1}}{d z^{2}}-k^{2} w_{1}=0
$$

From Eq. (24), we have

$$
w_{1}= \begin{cases}A_{+} \exp (-k z) & \text { for } z>z_{d}, \\ A_{-} \exp (k z) & \text { for } z<-z_{d},\end{cases}
$$

where $A_{+}$and $A_{-}$are arbitrary constants. From Eqs. (23) and (25), we have

$$
P_{1}=\mp \frac{i \rho_{g} \bar{\omega}}{k} w_{1} \text { at } z= \pm z_{d}
$$

Since $P_{1}$ should be continuous at $z= \pm z_{d}$, Eqs. (21) and (26) read

$$
\frac{d w_{1}}{d z}+\left( \pm k-\frac{1}{\bar{v}} \frac{d v_{0}}{d z}\right) w_{1}=0 \text { at } z= \pm z_{d}
$$

There are two types of solutions: (a) even solutions where $w_{1}(-z)=w_{1}(z)$, and (b) odd solutions where $w_{1}(-z)=$ $-w_{1}(z)$. It is revealed from our numerical calculations that even solutions are always stable. Thus, we show only the odd solutions in the following. In this case, the boundary conditions are

$$
\frac{d w_{1}}{d z}+\left(k-\frac{1}{\bar{v}} \frac{d v_{0}}{d z}\right) w_{1}=0 \text { at } z=z_{d},
$$

and

$$
w_{1}=0 \text { at } z=0,
$$

instead of Eq. (27). Further we restrict ourselves to the case where $k_{x}=0$, since this gives the most unstable mode for a given value of $k$. Numerical solutions of Eq. (22) with boundary conditions, Eqs. (28) and (29), will be shown in the next section. 


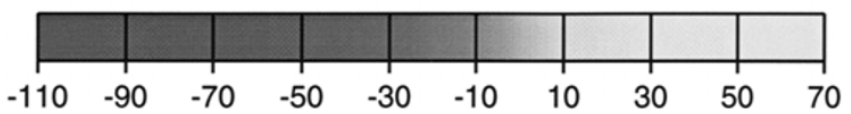

$$
\rho_{d 0}(0) / \rho_{g}=0.1 \quad J=0.1
$$

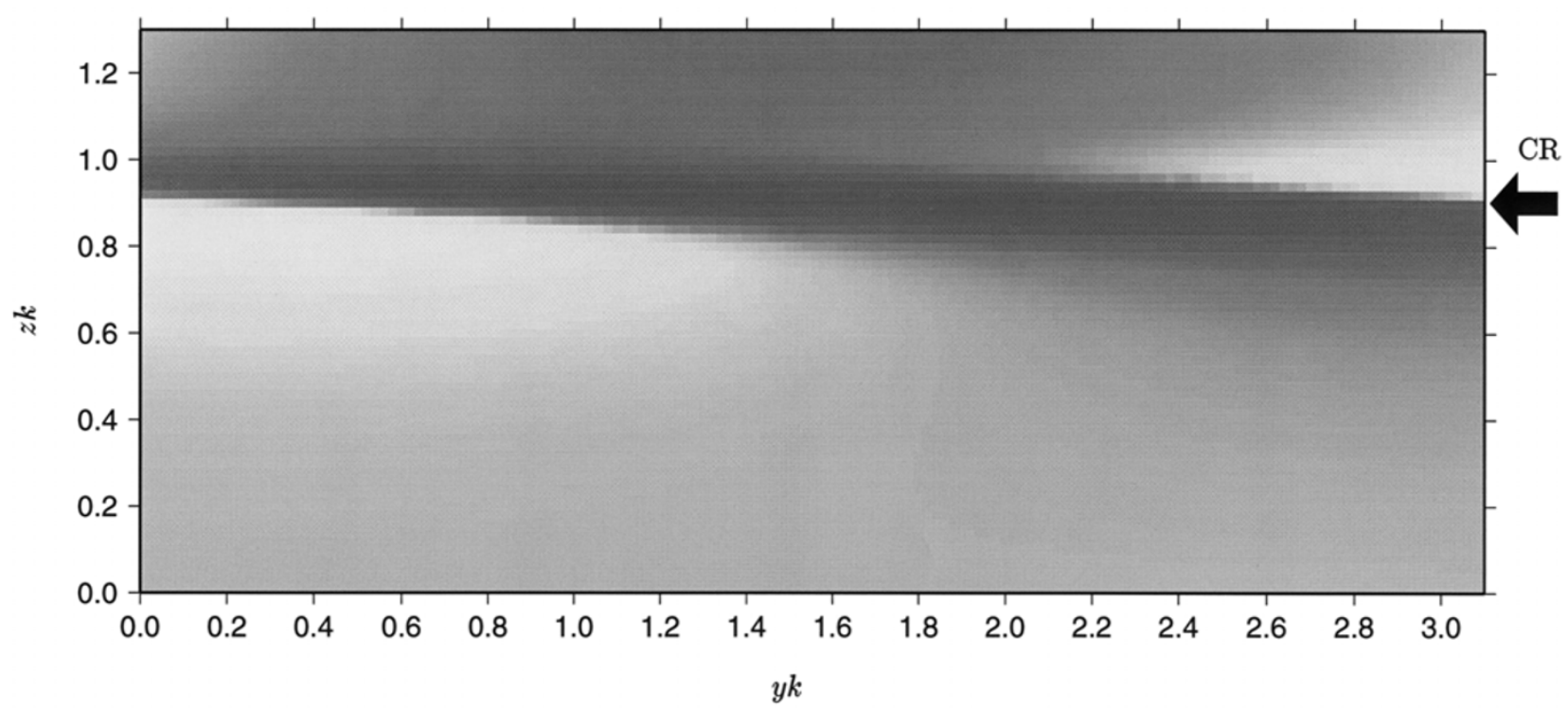

Fig. 14. The distribution of vorticity $\xi_{1} \equiv \partial_{y} w_{1}-\partial_{z} v_{1}$ of the mode with the most unstable wave number in the case where $J=0.1$ and $\rho_{d 0}(0) / \rho_{g}=0.1$. The co-rotation sheet is shown by the arrow with characters "CR".
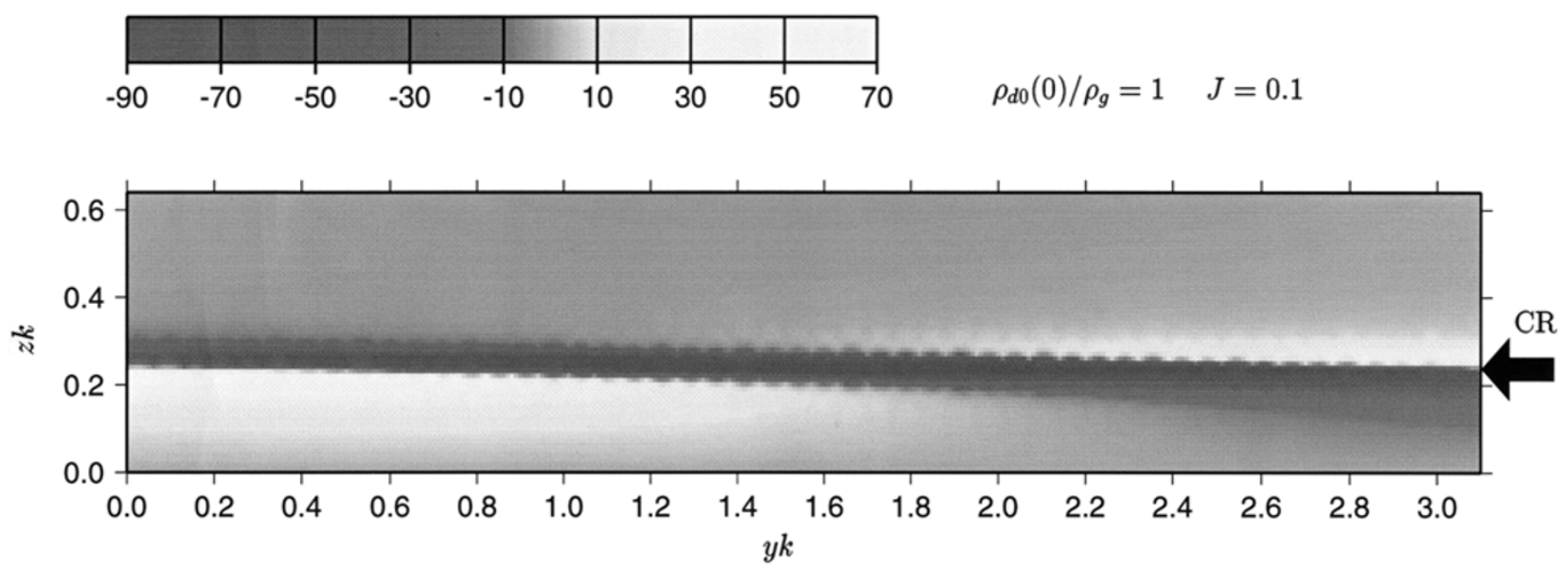

Fig. 15. Same as Fig. 14 except that $\rho_{d 0}(0) / \rho_{g}=1$.

\section{Numerical Results and Discussion}

An unperturbed state is characterized by two parameters: the Richardson number $J$ and the ratio of the dust density to the gas density on the midplane $\rho_{d 0}(0) / \rho_{g}$. Figures 1 to 3 show the dust density, the velocity and the velocity gradient, respectively, as functions of $z$, where the cases $\rho_{d 0}(0) / \rho_{g}=0.1,1$ and 10 with $J=0.1$ are drawn. As noted by Sekiya (1998), the characteristics of a density distribution given by Eq. (14) change considerably between the cases where $\rho_{d 0}(0) / \rho_{g}<<1$ and $\rho_{d 0}(0) / \rho_{g}>>1$. In the case $\rho_{d 0}(0) / \rho_{g}>>1$, a density distribution changes its character in the regions where $\rho_{d 0}(z) / \rho_{g}<<1$ and $\rho_{d 0}(z) / \rho_{g}>>1$, as seen in Fig. 1. Thus a velocity distribution also changes its character according to Eqs. (3) and (5), as seen in Figs. 2 and 3 , respectively.
In each case, we solved the perturbation equations numerically and obtained the growth rate of an unstable mode $\omega_{i}=\Im(\omega)$ as a function of the wave number $k$ (Fig. 4), where $\mathfrak{s}$ designates the imaginary part. The most unstable wave numbers are $\log \left(k^{2} \eta^{2} r^{2}\right)=1.989,0.730,0.849$ in the cases $\rho_{d 0}(0) / \rho_{g}=0.1,1$ and 10 , respectively, with $J=$ 0.1 . The reason why a mode has a peak growth rate will be discussed later.

Figures 5 to 7 show the growth rates of the mode with the most unstable wave number as functions of the Richardson number $J$ in cases $\rho_{d 0}(0) / \rho_{g}=0.1,1$ and 10 , respectively. As seen in these figures, the growth rate is much less than the Keplerian angular frequency as long as $J \gtrsim 0.1$, and the instability disappears when $J \gtrsim 0.22$. Thus the Coriolis force and the Keplerian shear which are neglected in this 


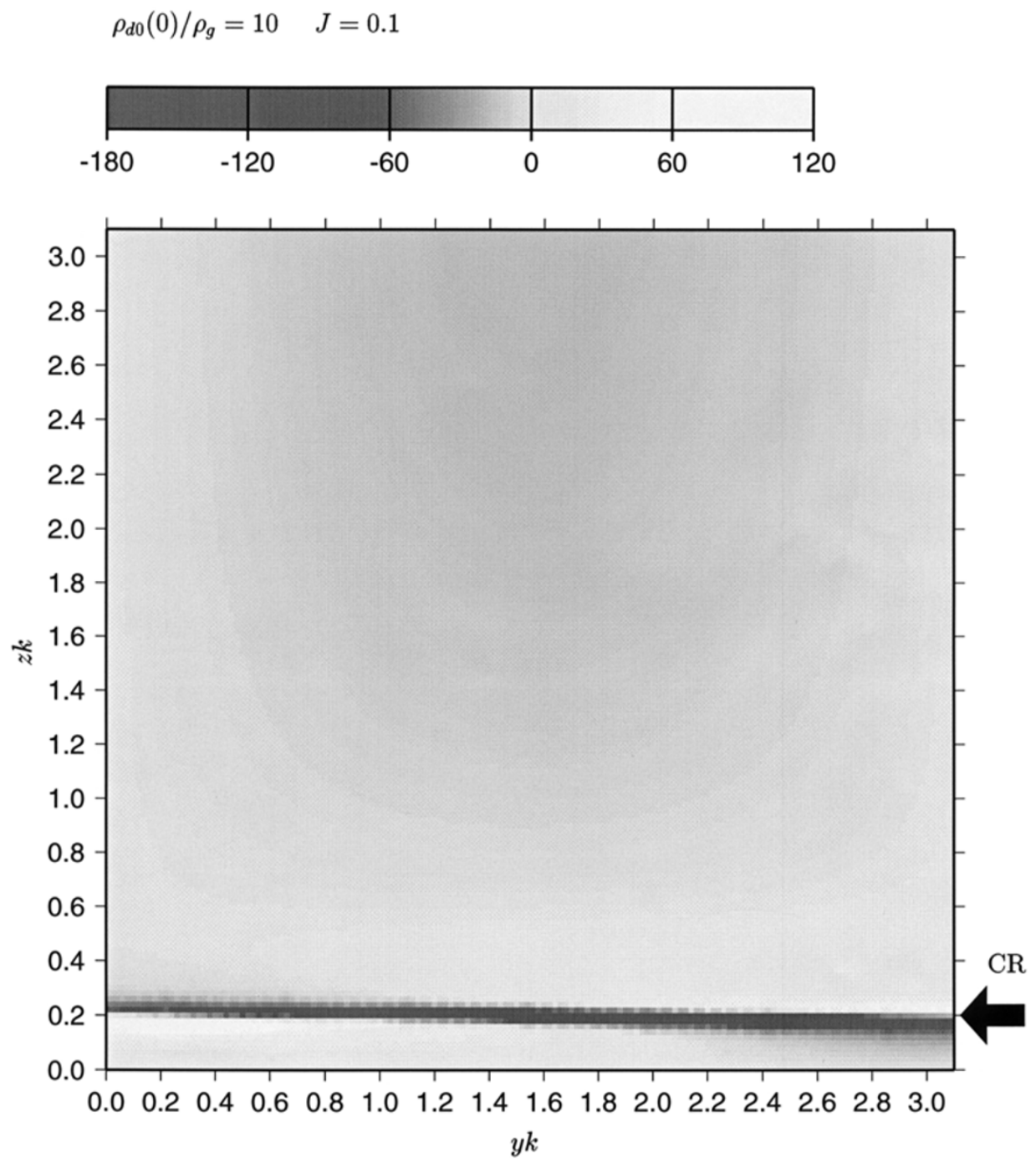

Fig. 16. Same as Fig. 14 except that $\rho_{d 0}(0) / \rho_{g}=10$.

paper and also in Sekiya (1998) may play a crucial effect on the instability. We plan to elucidate this problem in some subsequent papers.

In Figs. 8 to 10, the real and imaginary parts of $w_{1}$, which are the eigenfunctions of Eq. (22), are drawn in the cases where $\log \left(k^{2} \eta^{2} r^{2}\right)=0.1,1.989$ and 2.8 , respectively, with $J=0.1$ and $\rho_{d 0}(0) / \rho_{g}=0.1$. In Figs. 11 to 13 , the real and imaginary parts of $v_{1}$, which are calculated from Eq. (16) using the assumption $k_{x}=0$, are drawn, with the same parameters as Figs. 8 to 10, respectively. Hereafter we call a sheet, where the unperturbed flow velocity $v_{0}$ is equal to the pattern velocity of the perturbation $\Re(c)$, i.e. $\Re(\bar{v})=0$, a co-rotation sheet (or a co-rotation point in the sense of one dimensional calculation of $z$ ), where $\mathfrak{R}$ designates the real part. Note that Eq. (22) has a singularity at the co-rotation point if $c$ is real, i.e. for neutral stability. Although a solution is regular at the co-rotation point for a growing mode with $\Im(c)>0$, which we obtained here, an abrupt change of the behavior of a solution occurs around the co-rotation point as seen in Figs. 8 to 13, since the last three terms in parentheses of Eq. (22) have large values.

Figures 14 to 16 show the distributions of the vorticity $\xi_{1} \equiv \partial_{y} w_{1}-\partial_{z} v_{1}$ of the mode with the most unstable wave number in cases $\rho_{d 0}(0) / \rho_{g}=0.1,1$ and 10 , respectively, with $J=0.1$ (note that the unit is arbitrary, since we treat a linearized quantity). It is seen that large amplitudes of vorticity also distribute around a co-rotation sheet. These behavior around the co-rotation sheet (co-rotation point) is interpreted physically that the unperturbed quantities interact with the perturbed quantities around the co-rotation sheet (co-rotation point). A more detailed description of the corotation point (the critical point in usual terminology of the fluid mechanics) is seen in chapter 8 of Lin (1955).

As seen from Fig. 4, the growth rates have the maximum values at $\log \left(k^{2} \eta^{2} r^{2}\right)=1.989,0.730$ and 0.849 in the cases $\rho_{d 0}(0) / \rho_{g}=0.1,1$ and 10 , respectively with $J=0.1$. Here we consider the reason why a mode has a maximum growth rate at an intermediate value of $k$. Multiplying Eq. (20) by $\rho_{0} v_{1}^{*} / 2$ (where the superscript $*$ denotes the complex conjugate value) and taking the real part, we have

$$
2 \omega_{I} \frac{1}{4} \rho_{0}\left|v_{1}\right|^{2}=-\frac{1}{2} \rho_{0} \frac{d v_{0}}{d z} \Re\left(w_{1} v_{1}^{*}\right)+\frac{1}{2} k \Im\left(P_{1} v_{1}^{*}\right),
$$

(note that we assume $k_{x}=0$ and $k_{y}=k$ ). This equation shows that the azimuthal part of the perturbed kinetic energy is supplied by the shear $d v_{0} / d z$ and is transported through the 


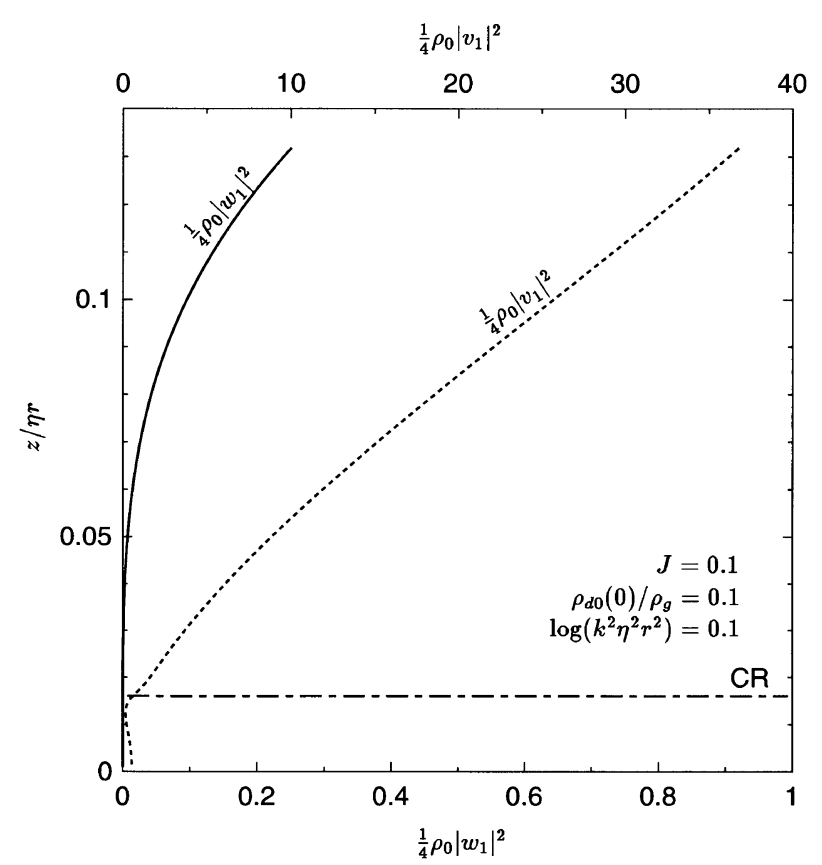

Fig. 17. The azimuthal and vertical parts of the perturbed kinetic energy density, $\rho_{0}\left|v_{1}\right|^{2} / 4$ and $\rho_{0}\left|w_{1}\right|^{2} / 4$, respectively, in the case where $\log \left(k^{2} \eta^{2} r^{2}\right)=0.1$, with $J=0.1$ and $\rho_{d 0}(0) / \rho_{g}=0.1$.

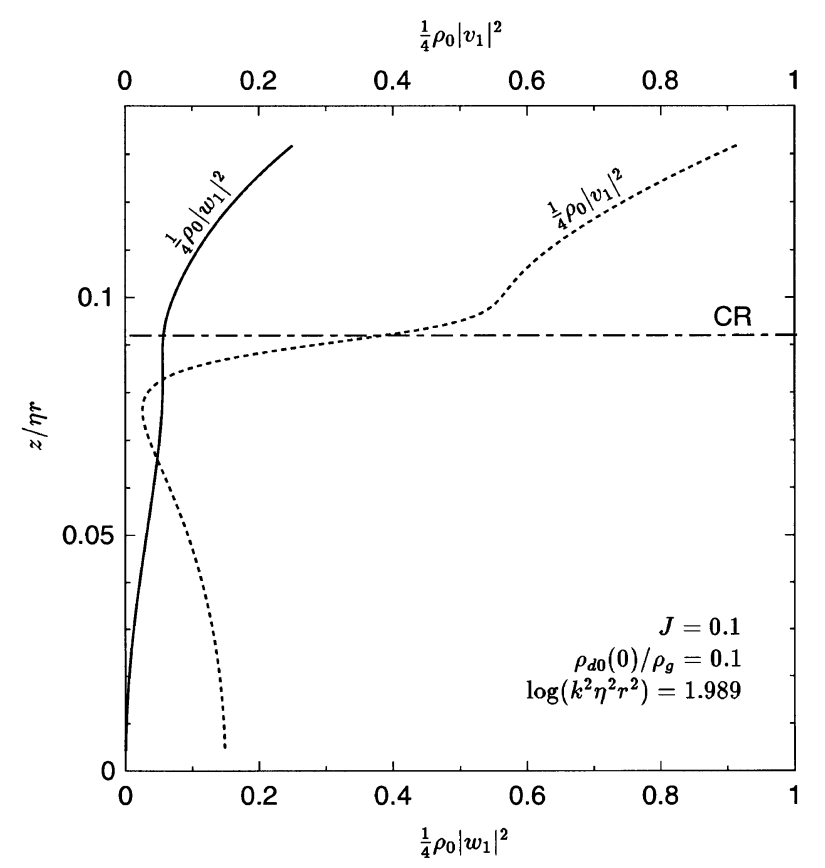

Fig. 18. Same as Fig. 17 except that $\log \left(k^{2} \eta^{2} r^{2}\right)=1.989$.

work done by the pressure perturbation (note that the phase average causes the extra factor $1 / 2$ in each term). Similarly, multiplying Eq. (20) by $\rho_{0} w_{1}^{*} / 2$ and taking the real part, we have

$$
2 \omega_{I} \frac{1}{4} \rho_{0}\left|w_{1}\right|^{2}=-\frac{1}{2} \Re\left(\frac{d P_{1}}{d z} w_{1}^{*}\right)-\frac{1}{2} \Omega_{K}^{2} z \Re\left(\rho_{1} w_{1}^{*}\right) .
$$

This equation shows that the vertical part of the perturbed kinetic energy is transported through work done by the pressure perturbation and is lost through the work done by the

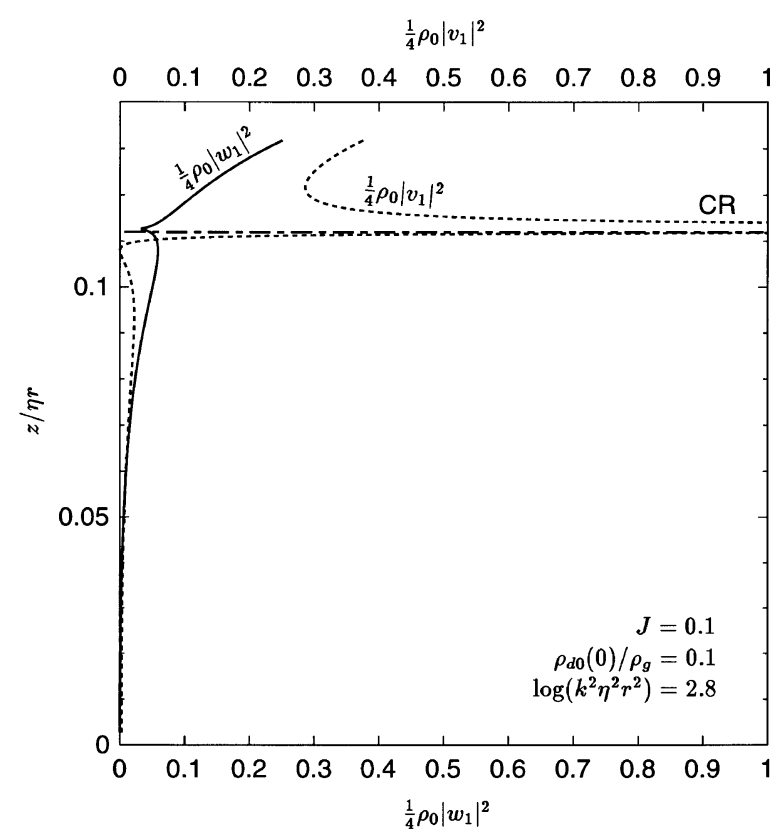

Fig. 19. Same as Fig. 17 except that $\log \left(k^{2} \eta^{2} r^{2}\right)=2.8$.

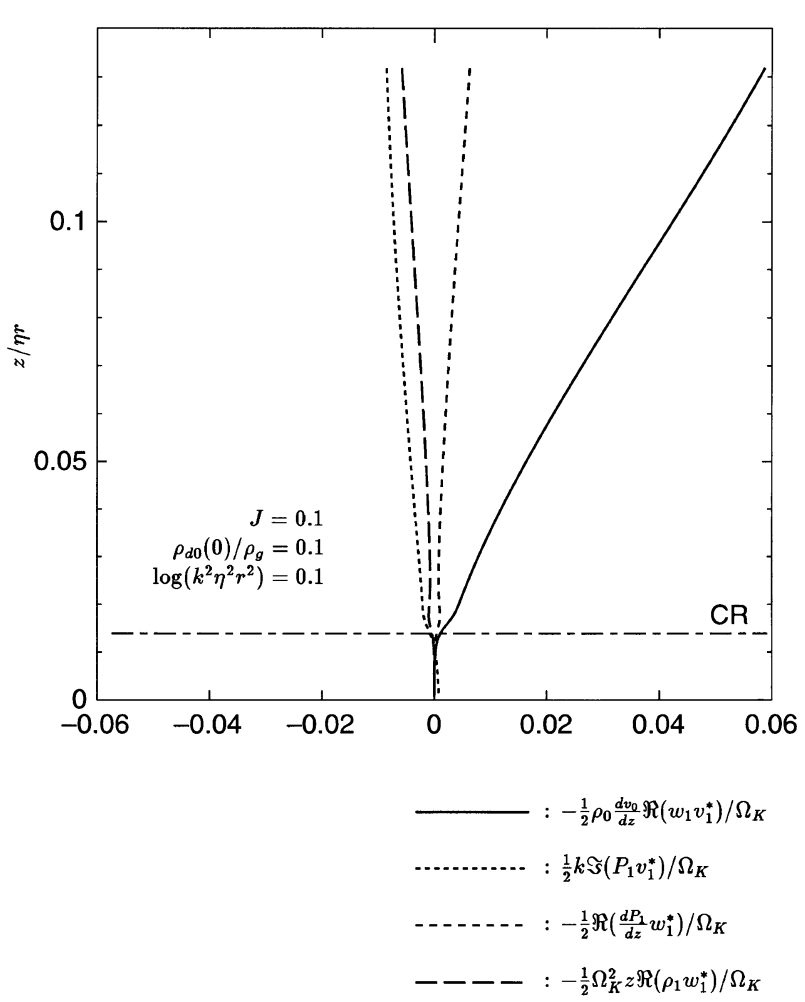

Fig. 20. Each term in the right hand sides of energy equations (30) and (31), in the case where $\log \left(k^{2} \eta^{2} r^{2}\right)=0.1$, with $J=0.1$ and $\rho_{d 0}(0) / \rho_{g}=0.1$.

$z$-component of the solar gravity $\Omega_{K}^{2} z$.

Figures 17 to 19 show the azimuthal and vertical parts of the perturbed kinetic energy density, with the same parameters as Figs. 8 to 10 , respectively. Figures 20 to 22 show each term in the right hand side of energy equations (30) and (31), with the same parameters as Figs. 8 to 10 , respectively. As $k \rightarrow 0,\left|w_{1}\right|<<\left|v_{1}\right|$ as seen from Fig. 17 [this figure shows the case where $\log \left(k^{2} \eta^{2} r^{2}\right)=0.1$ ], since 


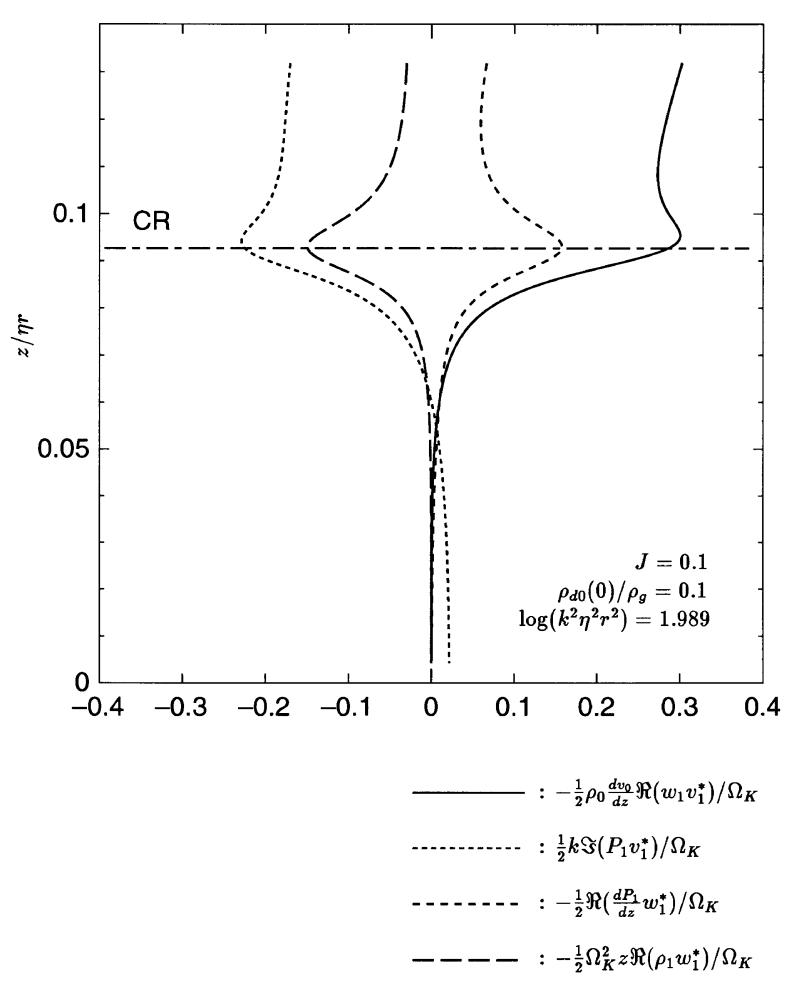

Fig. 21. Same as Fig. 20, except that $\log \left(k^{2} \eta^{2} r^{2}\right)=1.989$

$v_{1}$ increases due to Eq. (16) whereas the behavior of $w_{1}$ is not so strongly controlled by the value of $k$ as long as $k$ is small enough as seen from Eqs. (22) and (28). In this case, the energy input by shear $-\rho_{0}\left(d v_{0} / d z\right) \Re\left(w_{1} v_{1}^{*}\right) / 2 \Omega_{K}$ is small (see Fig. 20), to supply the large kinetic energy $\int\left[\rho_{0}\left(\left|w_{1}\right|^{2}+\left|v_{1}\right|^{2}\right) / 4\right] d z \sim \int\left(\rho_{0}\left|v_{1}\right|^{2} / 4\right) d z$ (see Fig. 17), since the former is linear and the latter is quadratic of $v_{1}$. In the case of large $k$ [e.g. $\log \left(k^{2} \eta^{2} r^{2}\right)=1.989$ and 2.8], the behavior of $w_{1}$ is strongly controlled by $k$ and the value of $\left|d w_{1} / d z\right|$ is nearly equal to $k\left|w_{1}\right|$ as seen from Eqs. (22) and (28). In this case $\left|w_{1}\right| \sim\left|v_{1}\right|$ as seen from Eq. (16) and also from Figs. 18 and 19. For such a large value $k$, an eigenvalue is determined by the continuation of the eigenfunction at the co-rotation point. In the case of $\log \left(k^{2} \eta^{2} r^{2}\right)=1.989$, values of $w_{1}$ and $d w_{1} / d z$ above and below the co-rotation point are similar (see Fig. 9) and there is no need to change the values abruptly around the co-rotation point to continue the eigenfunction. Thus Eq. (22) should not be nearly singular and a large value of $\Im(c)=\omega_{i} / k$ is allowed. As $k$ increases, the value of $d w_{1} / d z$ increases as seen from Eqs. (22) and (28), and the matching of values $w_{1}$ and $d w_{1} / d z$ above and below the co-rotation sheet become worse; thus an abrupt change of $w_{1}$ around the co-rotation point is needed to continue the function (see Fig. 10), and Eq. (22) should be nearly singular at the co-rotation point. Therefore $\Im(c)=\omega_{i} / k$ should be small.

\section{Conclusion}

The linear analysis of the shear induced instability of the dust layer in the solar nebula is made. In this paper, (1) the self-gravity is neglected; (2) the Coriolis force and the solar tidal force (thus the Keplerian shear) are also neglected; (3) the one-fluid model which is appropriate for small dust

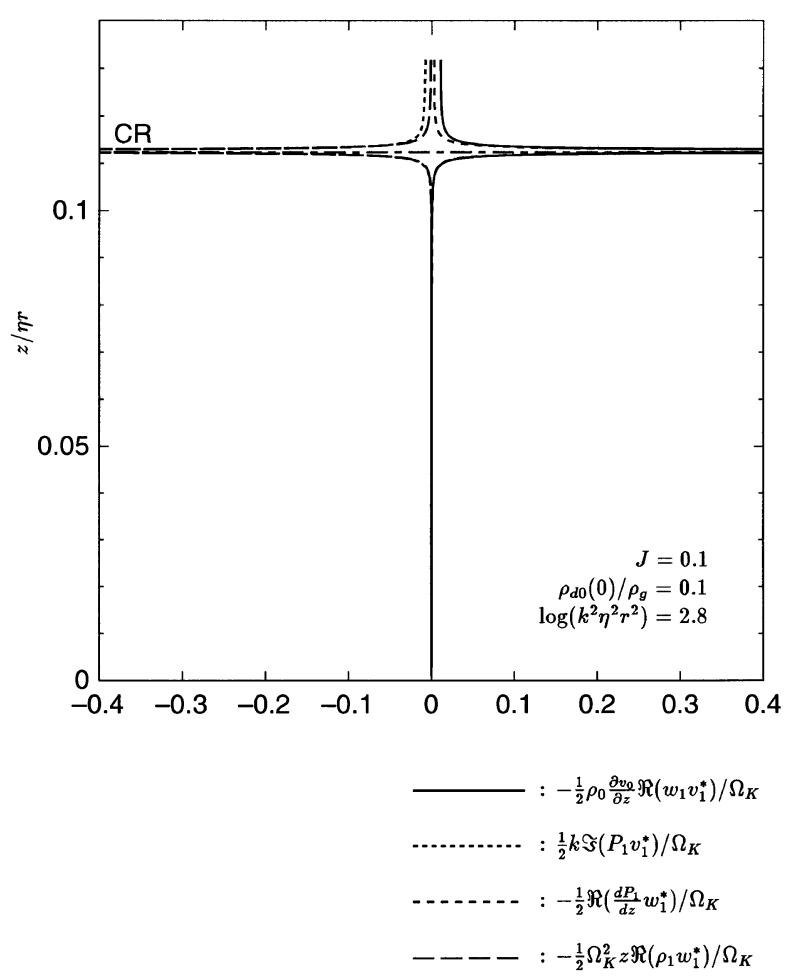

Fig. 22. Same as Fig. 20, except that $\log \left(k^{2} \eta^{2} r^{2}\right)=2.8$

aggregates is used. We solved a problem of non-rotating system without Keplerian shear $\partial v_{0} / \partial r$ but with shear in $z$ direction $\partial v_{0} / \partial z$. We have the following results: (A) The growth time is much longer than the Keplerian period, as long as the Richardson number $J \gtrsim 0.1$. (B) The instability disappears when $J \gtrsim 0.22$. Under the assumptions made in this paper, it is considered that the dust distribution in the solar nebula is well represented by Eqs. (14) and (4) with $J \approx 0.22$. Since this value is not so different from the critical Richardson number $J_{c}=0.25$ used by Sekiya (1998), the conclusion of Sekiya (1998), that the gravitational instability is inhibited in the nebula with the solar abundance, is correct as long as the above mentioned assumptions are valid. In cases where the dust sizes are much larger than $\sim \mathrm{cm}$, the two fluid model should be used to analyze the instability; this will be done in subsequent papers. The Coriolis force and the solar tidal force may also play essential roles; we also plan to treat this problem in subsequent papers.

Acknowledgments. We thank S. J. Weidenschilling and an anonymous reviewer for helpful remarks. Numerical calculations were performed partly on VX/1R and VX/4R at the Astronomical Data Analysis Center of the National Astronomical Observatory, Japan.

\section{References}

Champney, J. M., A. R. Dobrovolskis, and J. N. Cuzzi, A numerical turbulence model for multiphase flows in the protoplanetary nebula, Phys. Fluids, 7, 1703-1711, 1995.

Chandrasekhar, S., Hydrodynamic and Hydromagnetic Stability, 652 pp., Oxford Univ. Press, Oxford, 1961

Coradini, A., C. Federico, and G. Magni, Formation of planetesimals in an evolving protoplanetary disk, Astron. Astrophys., 98, 173-185, 1981.

Cuzzi, J. N., A. R. Dobrovolskis, and J. M. Champney, Particle-gas dynamics in the midplane of a protoplanetary nebula, Icarus, 106, 102-134, 1993.

Goldreich, P. and W. R. Ward, The formation of planetesimals, Astrophys. J., 183, 1051-1061, 1973. 
Howard, L. N., Note on a paper of John W. Miles, J. Fluid Mech., 10, 509-512, 1961.

Lin, C. C., The Theory of Hydrodynamic Stability, 155 pp., Cambridge Univ. Press, 1955.

Nakagawa, Y., K. Nakazawa, and C. Hayashi, Growth and sedimentation of dust grains in the primordial solar nebula, Icarus, 45, 517-528, 1981.

Nakagawa, Y., M. Sekiya, and C. Hayashi, Settling and growth of dust particles in a laminar phase of a low-mass solar nebula, Icarus, 67, 375390, 1986.

Safronov, V. S., Evolution of the Protoplanetary Cloud and Formation of the Earth and the Planets, 206 pp., Nauka, Moscow, [NASA Tech. Trans. F-677], 1969.

Sekiya, M., Gravitational instabilities in a dust-gas layer and formation of planetesimals in the solar nebula, Progr. Theor. Phys., 69, 1116-1130,
1983

Sekiya, M., Quasi-equilibrium density distributions of small dust aggregations in the solar nebula, Icarus, 133, 298-309, 1998.

Weidenschilling, S. J., Dust to planetesimals: Settling and coagulation in the solar nebula, Icarus, 44, 172-189, 1980.

Weidenschilling, S. J., Evolution of grains in a turbulent solar nebula, Icarus, 60, 553-567, 1984

Weidenschilling, S. J. and J. N. Cuzzi, Formation of planetesimals in the solar nebula, in Protostars and Planets III, edited by E. H. Levy and J. I. Lunine, pp. 1031-1060. Univ. of Arizona Press, Tucson, 1993.

M. Sekiya (e-mail: sekiya@geo.kyushu-u.ac.jp) and N. Ishitsu 